\title{
NUMERICAL AND EXPERIMENTAL INVESTIGATION INTO PROPULSION AND CAVITATION PERFORMANCE OF MARINE PROPELLER
}

\section{MARINE 2011}

\author{
NOBUHIRO HASUIKE ${ }^{*}$, SHOSABURO YAMASAKI ${ }^{*}$ AND JUN ANDO ${ }^{\dagger}$ \\ * Nakashima Propeller Co., Ltd. \\ 688-1, Joto-Kitagata, Higashi-ku, Okayama 709-0625, Japan \\ E-mail: nobuhiro@nakashima.co.jp, web page: http://www.nakashima.co.jp/ \\ ${ }^{\dagger}$ Faculty of Engineering, Kyushu University \\ 744 Motooka Nishi-ku Fukuoka 819-0395, Japan \\ E-mail: ando@nams.kyushu-u.ac.jp, Web page: http://www.eng.kyushu-u.ac.jp/e/index.html
}

Key words: CFD, Transitional flow, Cavitation, Pressure fluctuation

Summary. This paper discusses the application of the CFD to transitional and cavitating flow around marine propellers. Especially the emphasis is put on the adaption of the 3 equations turbulence model for the non-cavitating flow and tip vortex resolution on cavitating flow. This research finds 3 equations turbulence model is effective for prediction of the propeller open characteristics in the wide range of Reynolds number. Next, numerical simulation of cavitating flow with tip vortex resolution is validated. Furthermore, simple cavitation erosion index is applied and shows good agreement with experimental results. Finally, effect of tip vortex resolution on the pressure fluctuation at the top of the propeller is discussed.

\section{INTRODUCTION}

In the design of marine propeller, it is necessary for designers to achieve higher propeller open efficiency and good cavitation performance. Therefore, the higher accuracy prediction tool is expected for estimation of propeller open characteristics (POC in short), erosion risk and pressure fluctuation caused by cavitation at initial design stage.

For realizing high performance in full scale, it is necessary for propeller designers to estimate the scale effects of POC in higher accuracy. Further, in wake flow, pressure distribution on the blade surface and cavity void fraction effect on erosion risk and cavity volume time variation gratefully effects on pressure fluctuation amplitude. Therefore, numerical tool which gives detail flow characteristics of cavitating flow is highly expected.

In this paper, newly developed 3 equations $\mathrm{k}-\mathrm{k}_{\mathrm{L}}-\omega$ model is applied to the transitional flow around model propellers and compared with other typical turbulence models and experimental results. Secondly, POC in the wide range of Reynolds number between model and full-scale is predicted and the boundary layer characteristics and variation of propeller efficiency is discussed.

Further, detailed cavitation simulation including tip vortex cavitation is conducted. Simple 
cavitation erosion index is introduced and validated with high speed video observation and paint erosion test result.

Finally, simulated pressure fluctuation and variation of cavity volume is compared with the experimental results and the effectiveness of numerical simulation is investigated.

\section{NUMERICAL MODEL}

In this research, transitional and cavitating flow around propeller were simulated using SOFTWARE CRADLE SCRYU/Tetra V9 software, which was based on a finite volume method with an unstructured grid. The k- $\varepsilon$ model, the Shear-Stress Transport k- $\omega$ model ${ }^{1,2}$ were applied to the transitional flow. In addition to these widely used turbulence models, newly developed 3-eytations $\mathrm{k}-\mathrm{k}_{\mathrm{L}}-\omega$ model was also applied. For the non-uniform cavitating flow simulations, the $\mathrm{k}-\varepsilon$ model and the full-cavitation model was applied and the simple erosion index was introduced.

\subsection{Transitional flow simulation}

It is important to predict the transition point of a flow around a propeller in operating in low-Reynolds-number. LKE (Laminar Kinetic Energy) model ${ }^{3}$ (Walters \& Leylek 2004) was developed to simulate the transitional flow.

In the LKE model, the disturbance energy in a pre-transitional region of a boundary layer is represented as Laminar Kinetic Energy $\left(k_{L}\right)$, while the turbulence energy is as $k$. The transport equation of $k_{L}$ is solved by using two equations of fully turbulent model. SC/Tetra introduces the following $\mathrm{k}-\mathrm{k}_{\mathrm{L}}-\omega$ model $^{4}$ (Walters \& Cokljat 2008) which was developed based on the k- $\omega$ modec:

$$
\begin{aligned}
\frac{\partial \rho k_{T}}{\partial t}+\frac{\partial \rho u_{i} k_{T}}{\partial x_{i}} & =\rho\left(P_{k_{T}}+R_{B P}+R_{N A T}-\omega k_{T}-D_{T}\right)+\frac{\partial}{\partial x_{i}}\left(\left(\mu+\frac{\rho \alpha_{T}}{\sigma_{k}}\right) \frac{\partial k_{T}}{\partial x_{i}}\right) \\
\frac{\partial \rho k_{L}}{\partial t}+\frac{\partial \rho u_{i} k_{L}}{\partial x_{i}} & =\rho\left(P_{k_{L}}-R_{B P}-R_{N A T}-D_{L}\right)+\frac{\partial}{\partial x_{i}}\left(\mu \frac{\partial k_{L}}{\partial x_{i}}\right) \\
\frac{\partial \rho \omega}{\partial t}+\frac{\partial \rho u_{i} \omega}{\partial x_{i}} & =\rho\left[C_{\omega 1} \frac{\omega}{k_{T}} P_{k_{T}}+\left(\frac{C_{\omega R}}{f_{W}}-1\right) \frac{\omega}{k_{T}}\left(R_{B P}+R_{N A T}\right)-C_{\omega 2} f_{W}^{\frac{4}{3}} \omega^{2}+f_{\omega} \alpha_{T} f_{W}{ }^{2} \frac{\sqrt{k_{T}}}{Y^{3}}\right] \\
& +\frac{\partial}{\partial x_{i}}\left(\left(\mu+\frac{\rho \alpha_{T}}{\sigma_{\omega}}\right) \frac{\partial \omega}{\partial x_{i}}\right)
\end{aligned}
$$

The parameter $P_{k T}$ and $P_{k L}$ are both production terms of $k_{T}$ and $k_{L}$.

The parameter $v_{T, S}$ and $v_{T, l}$ are the eddy viscosities of small scale and large scale, respectively. The sum of these values $\left(v_{t}=v_{T, S}+v_{T, l}\right.$ is used for the eddy viscosity of the momentum equation. The parameter $R_{B P}$ and $R_{N A T}$ are the contributions of the bypass transition and the natural transition, respectively. The contribution of the bypass transition increases as the turbulent intensity in the external flow increases.

\subsection{Cavitating flow simulation}

The full-cavitation model ${ }^{5}$ (Singhal et al. 2002) accounts for all first-order effects, i.e., phase change, bubble dynamics, turbulent pressure fluctuations and non-condensable gases contained in the liquid.

The fluid density is a function of vapor mass fraction $f$, which is computed by solving a 
transport equation coupled with the mass and momentum conservation equations. Suffixes $v$, $g, l$ denote the vapor phase, non-condensable gas (NCG in short), liquid respectively. $\vec{v}$ is velocity vector. $\gamma$ is diffusion coefficient of the vapor mass fraction. Net phase change rate is expressed as $R_{e}-R_{c}$. The source terms $R_{e}$ and $R_{c}$ denote vapor evaporation and condensation rates. Where, $k$ and $\sigma$ are the turbulent kinetic energy and the surface tension. The phase change rate $R_{e}$ and $R_{c}$ are derived from the Rayleigh-Plesset equation and limiting bubble size (interface surface area per unit volume of vapor) is considered. $C_{e}$ and $C_{c}$ are the empirical components. It is assumed that saturated vapor pressure $P_{v}$ depends on the turbulence kinetic energy. ((8), (9)) Subscripts turb denotes the turbulence.

$$
\begin{aligned}
& \frac{1}{\rho}=\frac{f_{v}}{\rho_{v}}+\frac{f_{g}}{\rho_{g}}+\frac{1-f_{v}-f_{g}}{\rho_{l}} \\
& \frac{\partial}{\partial t}(\rho f)+\nabla \cdot(\rho \vec{v} f)=\nabla \cdot(\gamma \nabla f)+R_{e}-R_{c} \\
& R_{e}=C_{e} \frac{\sqrt{k}}{\sigma} \rho_{l} \rho_{v} \sqrt{\frac{2\left(p_{v}-p\right)}{3 \rho_{l}}}\left(1-f_{v}-f_{g}\right) \\
& R_{c}=C_{c} \frac{\sqrt{k}}{\sigma} \rho_{l} \rho_{l} \sqrt{\frac{2\left(p-p_{v}\right)}{3 \rho_{l}}} f_{v} \\
& P_{v}=\left(P_{\text {sat }}+\frac{P_{\text {turb }}^{\prime}}{2}\right) \\
& P_{\text {turb }}^{\prime}=0.39 \rho k
\end{aligned}
$$

\subsection{Numerical grids}

The computational domain is composed of the inner rotational part including the propeller and the outer stationary part. The stationary part and the rotational part are connected discontinuously. Constant velocity and zero pressure are prescribed at the inlet and the outlet boundary. Fig. 1 shows the computational domain. The numerical mesh is an unstructured grid, and basic cells are tetrahedral and prismatic cells are applied to near the blade surface for resolving the boundary layer (Fig. 2). The first layer thickness of the prism layer was set to a non-dimensional wall distance for a wall-bounded flow $\left(y^{+}\right.$in short $)=50$ in the case of the k- $\varepsilon$ model. $y^{+}=50$ was set in the case of the SST $\mathrm{k}-\omega$ model and the $\mathrm{k}-\mathrm{k}_{\mathrm{L}}-\omega$ model. In this research, fine meshes are applied near the tip region for the resolution of tip vortex(Fig. 3).

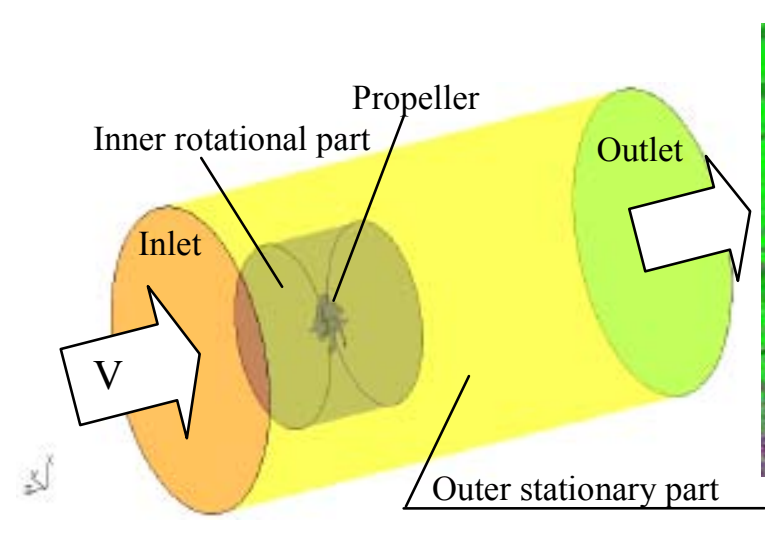

Figure 1: Computational domain

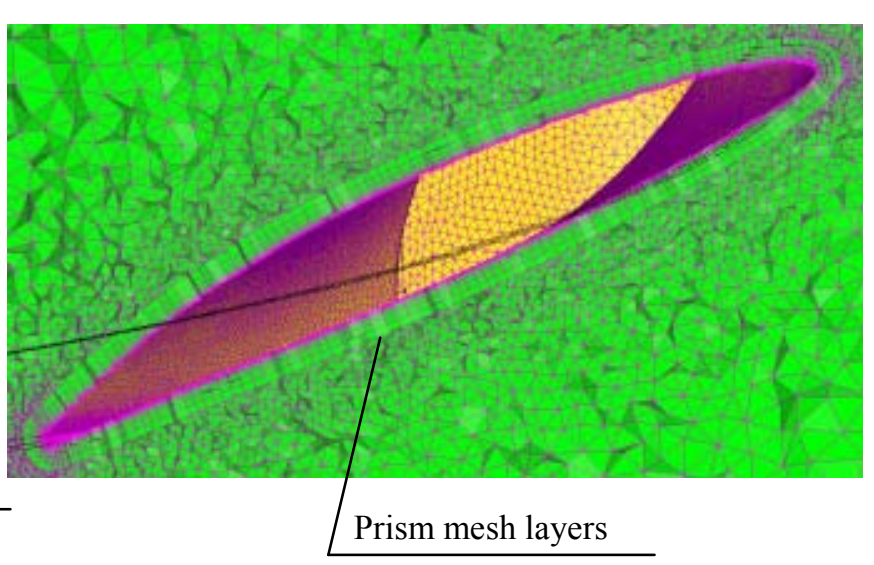

Figure 2: Prism mesh arrangement near blade surface 


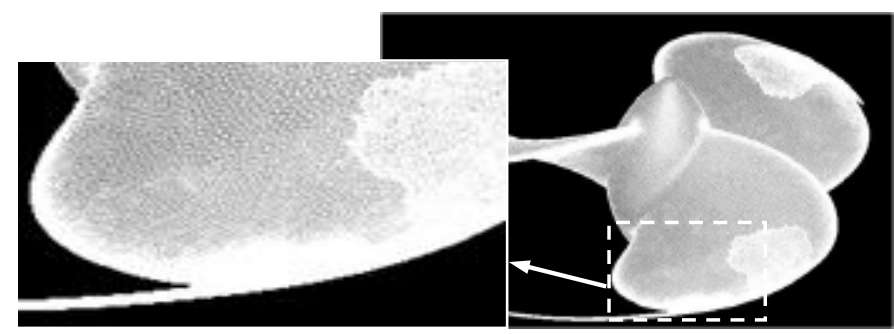

Figure 3: Mesh near the blade surface

\section{NUMERICAL SIMULATION OF PROPELLER OPEN CHARACTERISTICS}

\subsection{Propeller open characteristics}

POC at model scale gratefully depends on mixture ratio of laminar and turbulent flow. DTMB P4119 propeller, ${ }^{6,7}$ was selected for the benchmark. This propeller's experimental dataset was used as 22nd ITTC benchmark of RANS calculations. Table 1 and Fig. 4 show the principal particulars and propeller shape. The k- $\varepsilon$ model, the SST k- $\omega$ model with low Reynolds correction ${ }^{2}$ (SST k- $\omega$ (Low) in short), the SST k- $\omega$ model without low Reynolds correction(SST $\mathrm{k}-\omega\left(\mathrm{w} / \mathrm{o}\right.$ corr.) in short) and the $\mathrm{k}-\mathrm{k}_{\mathrm{L}}-\omega$ model were applied. Turbulent intensity $T_{\text {uin }}$ at inlet was set to $1 \%$ for the k- $\varepsilon$ model and the SST k- $\omega$ models and set to $5 \%$ for the $\mathrm{k}-\mathrm{k}_{\mathrm{L}}-\omega$ model.

First of all, POC in a different operation point is compared in Fig. 5. In the case of the k- $\varepsilon$ model, thrust coefficient $\mathrm{K}_{\mathrm{T}}$ was overall small compared with the experiment, and torque constant $\mathrm{K}_{\mathrm{Q}}$ was large excluding advancement ratio $\mathrm{J}=1.1$. The SST $\mathrm{k}-\omega$ (Low) predicted higher $\mathrm{K}_{\mathrm{T}}$ and corresponding $\mathrm{K}_{\mathrm{Q}}$ value compared with experiment. Predicted open efficiency $\eta_{\mathrm{o}}$ was overestimated at design point $\mathrm{J}=0.833$. On the other hand, The $\mathrm{k}-\mathrm{k}_{\mathrm{L}}-\omega$ model gave corresponding $\mathrm{K}_{\mathrm{T}}$ and little smaller $\mathrm{K}_{\mathrm{Q}}$ value. Propeller open efficiency $\eta_{\mathrm{o}}$ was more corresponding well than the other turbulence models at design point $\mathrm{J}=0.833$.

Next, the velocity distributions in boundary layer are compared in Fig. 6. $Y / C$ and $V_{B} / V_{R}$ denote non-dimensional distance from wall and velocity in the boundary layer divided by outer flow respectively. The $\mathrm{k}-\varepsilon$ model shows good agreement with the experimental measurement results shown in figure as "Tripped" which means the state of with roughness on the leading edge of the propeller. The estimated boundary layer thickness by the SST k- $\omega$ (Low) model is thinner than the experimental measurement results shown in figure as "Smooth" which means the state of without roughness on the leading edge of the propeller. The $\mathrm{k}-\mathrm{k}_{\mathrm{L}}-\omega$ model gave better result than the SST k- $\omega$ (Low) in case of "Smooth".

Turbulence kinetic energy distributions around the blade section at $70 \%$ radius are compared in Figs. 7(a)-(d). The k- $\varepsilon$ and the SST k- $\omega$ (w/o corr.) predicted the fully turbulent flow. Underestimation of $\eta_{\mathrm{o}}$ was mainly due to overestimation of viscous component of $\mathrm{K}_{\mathrm{Q}}$. On the other hand, the flow field simulated by the SST k- $\omega$ (Low) was fully laminar and which causes underestimation of viscous component of $\mathrm{K}_{\mathrm{Q}}$. In the case of the $\mathrm{k}-\mathrm{k}_{\mathrm{L}}-\omega$ model, flow field was mainly composed by laminar flow and partially composed by turbulent flow. In propeller open water test, leading edge roughness was not applied. Therefore, the $\mathrm{k}-\mathrm{k}_{\mathrm{L}}-\omega$ model gave more corresponding $\eta_{\mathrm{o}}$ for the POC prediction at model scale. 
Table 1 Principal particulars of DTMB P4119 propeller ${ }^{6,7}$

\begin{tabular}{lc}
\hline & DTMB P4119 \\
Number of blades & 3 \\
Diameter & $0.3048 \mathrm{~m}$ \\
Pitch ratio(0.7R) & 1.084 \\
Skew angle & $0^{\circ}$ \\
\hline
\end{tabular}
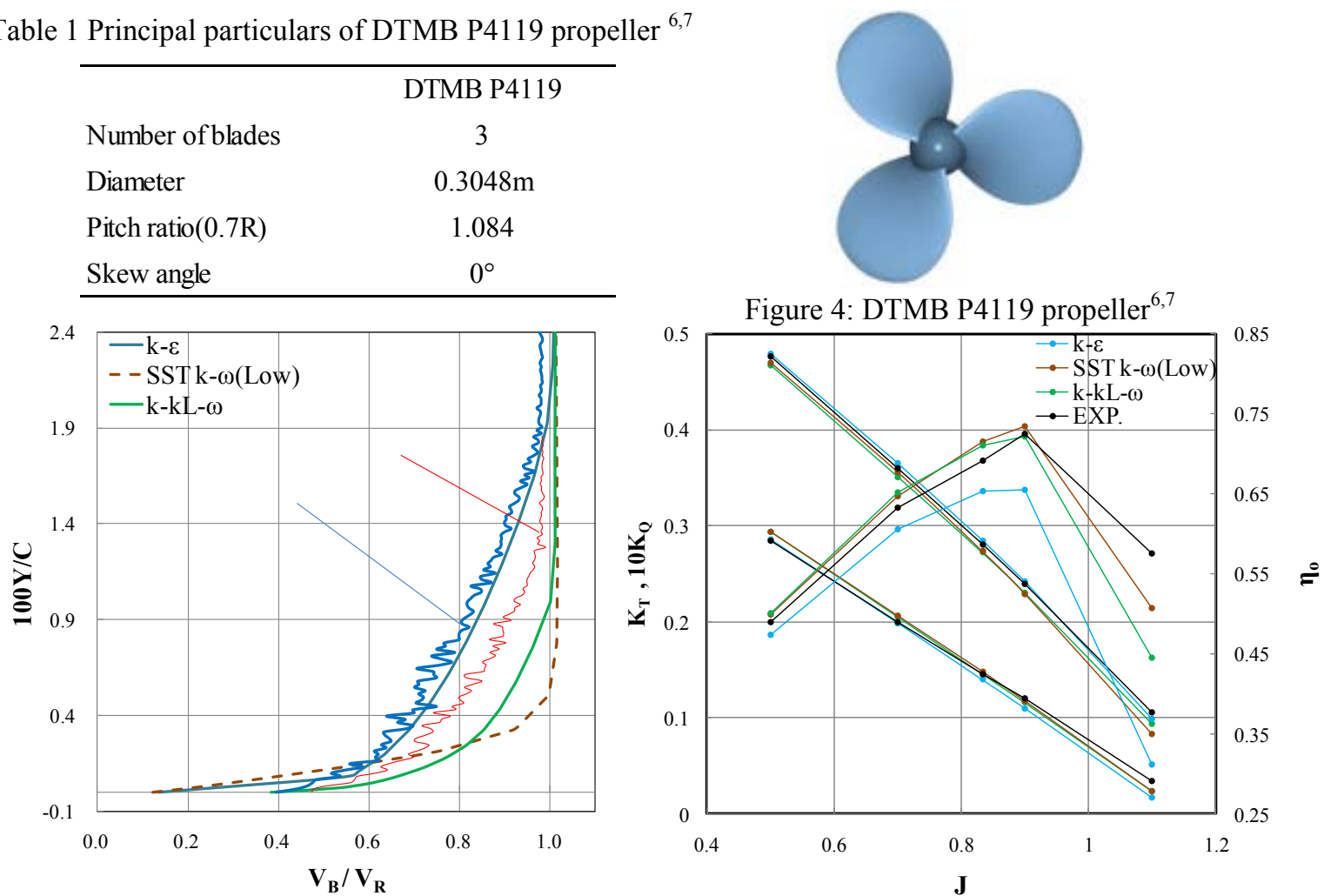

Figure 6 : Boundary layer profiles at $\mathrm{r} / \mathrm{R}=0.7$, back side, $\mathrm{x} / \mathrm{C}=0.9 \quad$ Figure 5: Propeller open characteristics

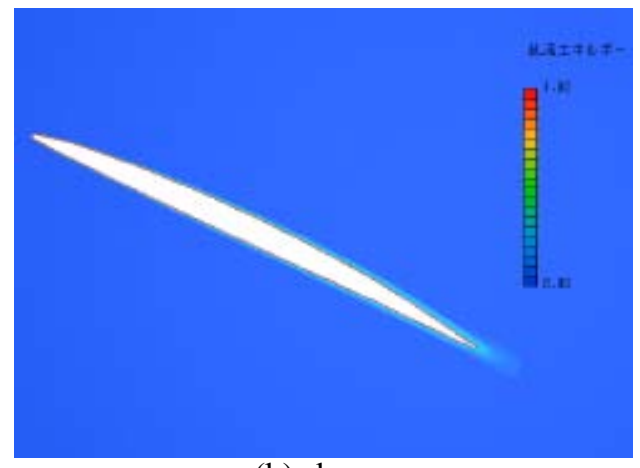

(b) $\mathrm{k}-\varepsilon$

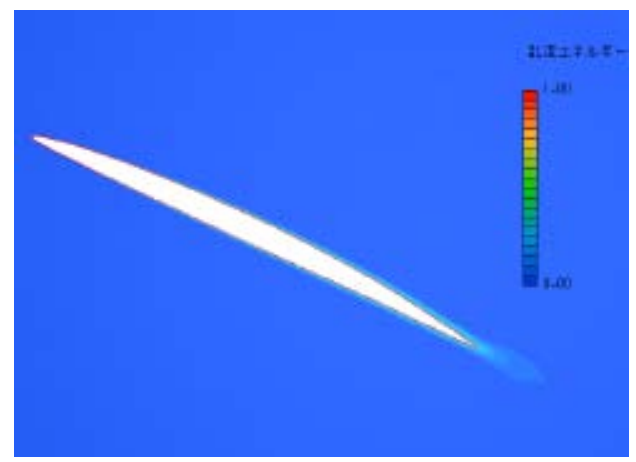

(c) SST k- $\omega$ w/o.corr

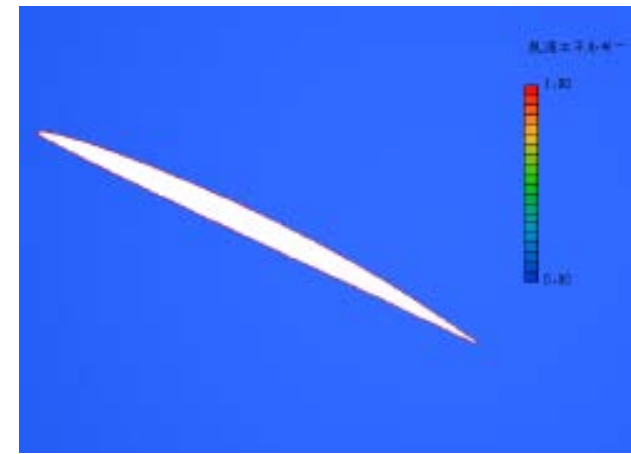

(a) SST k- $\omega$ Low

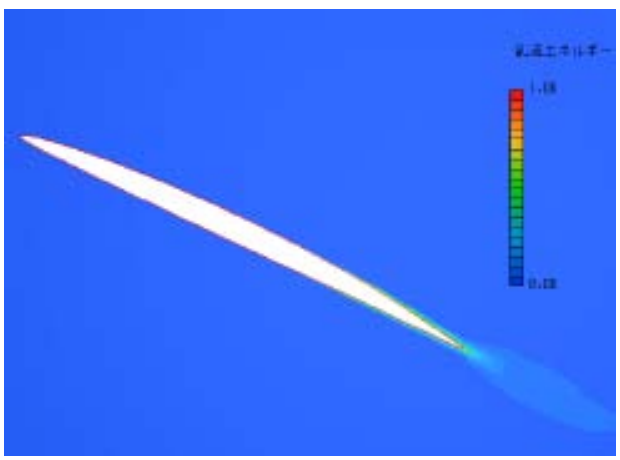

(d) $\mathrm{k}-\mathrm{k}_{\mathrm{L}}-\omega$

Figure 7: Turbulent kinetic energy at $\mathrm{r} / \mathrm{R}=0.7$ 


\subsection{Transitional flow simulation}

Prediction accuracy of the transitional position by using the $\mathrm{k}-\mathrm{k}_{\mathrm{L}}-\omega$ model was validated with experimental results. MP2293R propeller has 'MAU' blade section which was traditionally used in past decades. MP0193R has laminar flow type of blade section. Principal particulars of propellers are shown in Table 2. Experimental and simulated results are shown in Fig. 8 and 9. Back side transitional line was located at about $60 \%$ chord length from leading edge in case of MP2293 and located at about 70\% in case of MP0193. Further, face side oil flow pattern was largely different among these two propellers. Simulated results of both MP2293R and MP0193 show good agreement with experimental results. It was confirmed that the $\mathrm{k}-\mathrm{k}_{\mathrm{L}}-\omega$ model predicted the effects of blade section on transitional region.

Table 2 Principal particulars of model propellers

\begin{tabular}{lll}
\hline & \multicolumn{2}{l}{ MP2293R MP0193R } \\
Number of blades & 5 & 5 \\
Diameter & $250 \mathrm{~mm}$ & $250 \mathrm{~mm}$ \\
Pitch ratio(0.7R) & 0.703 & 0.7506 \\
Expanded area ratio & 0.4 & 0.4 \\
Skew angle & $20^{\circ}$ & $20^{\circ}$ \\
\hline
\end{tabular}

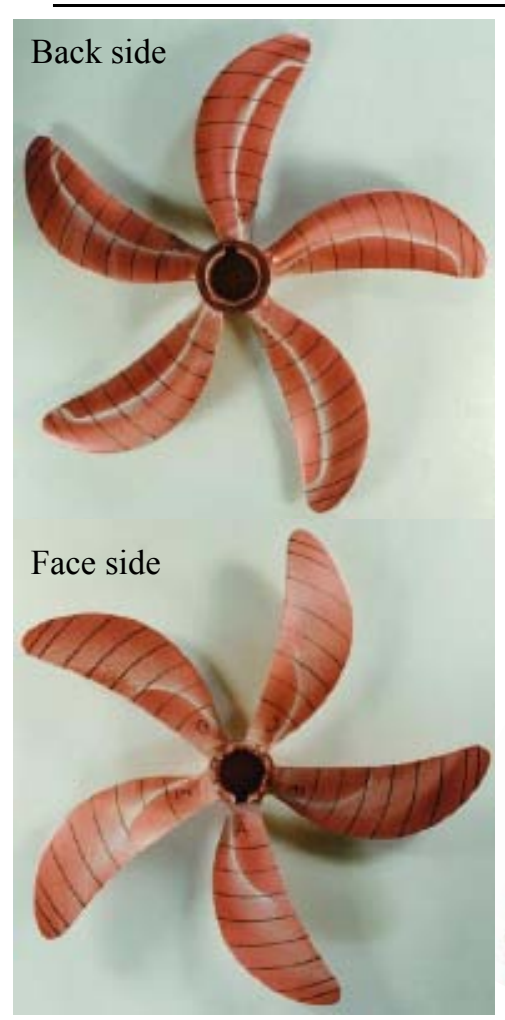

Experiment

(Oil Flow Visualization)

Figure 8: Surface streamlines of MP2293

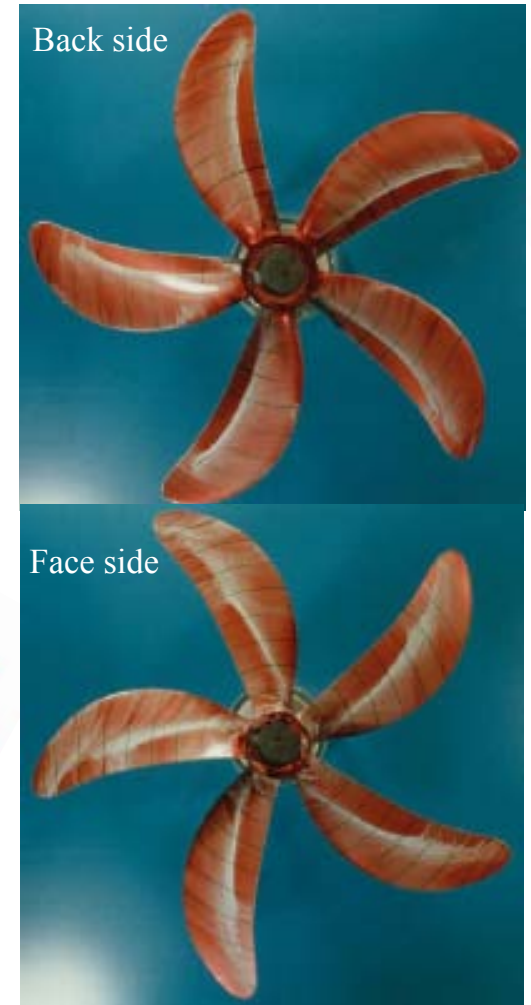

Experiment (Oil Flow Visualization)
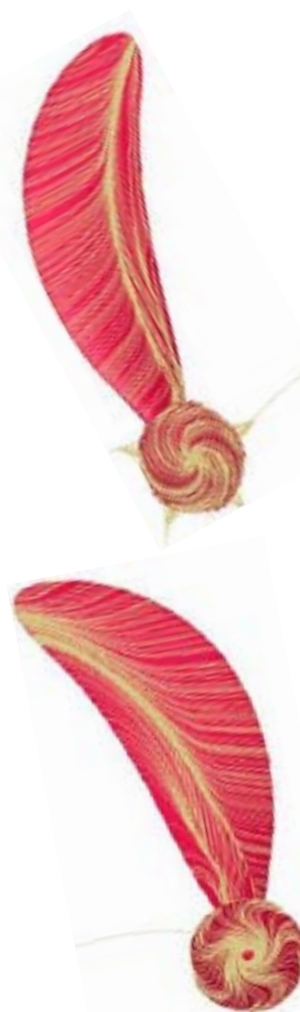

Calculation (Limiting Streamlines)
Figure 9: Surface streamlines of MP0193 


\subsection{Scale effects of propeller open characteristics}

Seiun-Maru conventional model propellers(CP), which experimental data includes open water test results in the wide range of Reynolds number were selected for the calculation. Experimental results were referred from Funeno's literature ${ }^{8}$. Calculation results are compared with experimental results in Figs. 10-12. Subscripts $p$ and $v$ denote pressure component and viscous component respectively. Fig. 13 shows typical representative of flow pattern. Turbulent flow is located at outer side of critical radius (C.R.).

1) SST k- $\omega$ (w/o corr.), SST k- $\omega$ (Low), $\mathrm{T}_{\text {uin }}=5 \%$

Simulated flow field was fully turbulent. Pressure component of $\mathrm{K}_{\mathrm{T}}$ increased and frictional component of $\mathrm{K}_{\mathrm{T}}$ decreased when Reynolds number increased. Pressure component of $\mathrm{K}_{\mathrm{Q}}$ increased and frictional component of $\mathrm{K}_{\mathrm{Q}}$ decreased when Reynolds number increased. $\mathrm{K}_{\mathrm{Q}}$ much depended on frictional component and decreased totally. As a result, $\eta_{\mathrm{o}}$ simply increased, however $\eta_{\mathrm{o}}$ was lower than experimental results at lower range of Reynolds number because of the overestimation of the frictional component. .

2) SST k- $\omega$ (Low), $\mathrm{T}_{\text {uin }}=1 \%$

Simulated flow field was mixture of laminar and turbulent flow and its critical radius was fixed in the range of $\mathrm{R}_{\mathrm{n}}(\mathrm{K})=2.5 \times 10^{5} \sim 3.7 \times 10^{5}$. In this range of Reynolds number, $\eta_{\mathrm{o}}$ increased by decrease of boundary layer thickness. In the range of $R_{n}(K)=3.7 \times 10^{5} \sim 1 \times 10^{6}$, critical radius moved toward inner radius and turbulent area increased when Reynolds number increased. $\eta_{\mathrm{o}}$ decreased by increasing wall shear stress of turbulent flow part. Over the range of $R_{n}(K)=1 \times 10^{6}$, flow field was fully turbulent. By decreasing of turbulent boundary layer thickness, $\eta_{\mathrm{o}}$ increased at this range of Reynolds number.

3) $\mathrm{k}-\mathrm{k}_{\mathrm{L}}-\omega$ model, $\mathrm{T}_{\text {uin }}=5 \%$

Simulated flow field was mixture of laminar and turbulent flow and its critical radius was fixed in the range of $\mathrm{R}_{\mathrm{n}}(\mathrm{K})=2.5 \times 10^{5} \sim 1 \times 10^{6}$. In this range of Reynolds number, $\eta_{\mathrm{o}}$ increased by decrease of boundary layer thickness. This tendency was corresponding to propeller open water test. In the range of $R_{n}(K)=1 \times 10^{6} \sim 3 \times 10^{6}$, critical radius moved toward inner radius and turbulent area increased when Reynolds number increased. $\eta_{\mathrm{o}}$ decreased by increasing wall shear stress of turbulent flow part. Over the range of $R_{n}(K)=3 \times$ $10^{6}$, flow field was fully turbulent.

Table 3 Principal particulars of model propellers

\begin{tabular}{ll}
\hline Number of blades & 5 \\
Diameter & $400(950) \mathrm{mm}$ \\
Pitch ratio & 0.95 \\
Expanded area ratio & 0.65 \\
Skew angle & $10.5^{\circ}$ \\
\hline
\end{tabular}

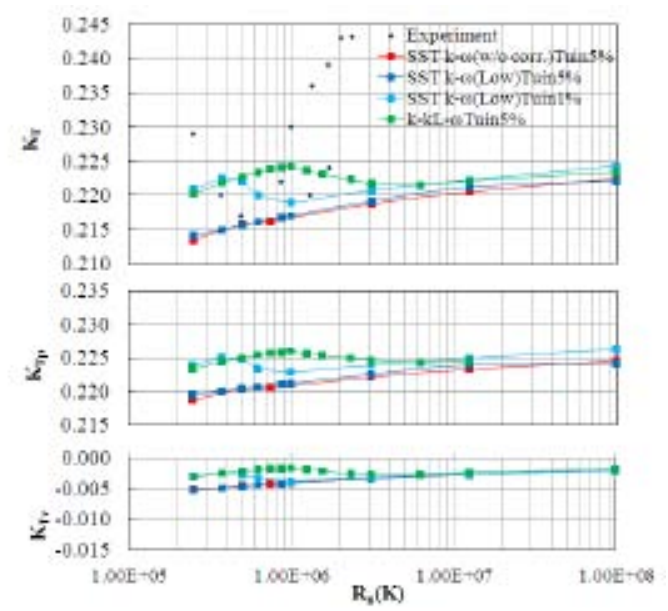

Figure 10: Effect of Reynolds number on $\mathrm{K}_{\mathrm{T}}$ 


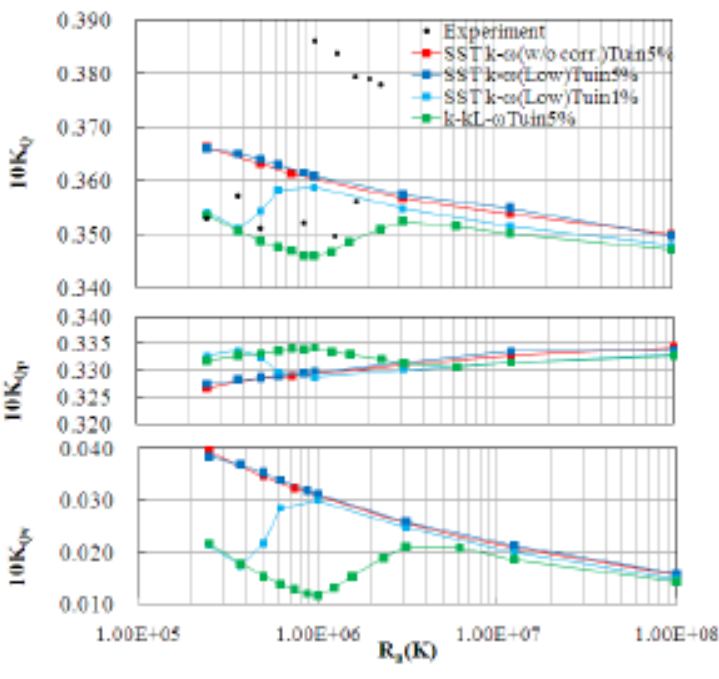

Figure 11: Effect of Reynolds number on $\mathrm{K}_{\mathrm{Q}}$

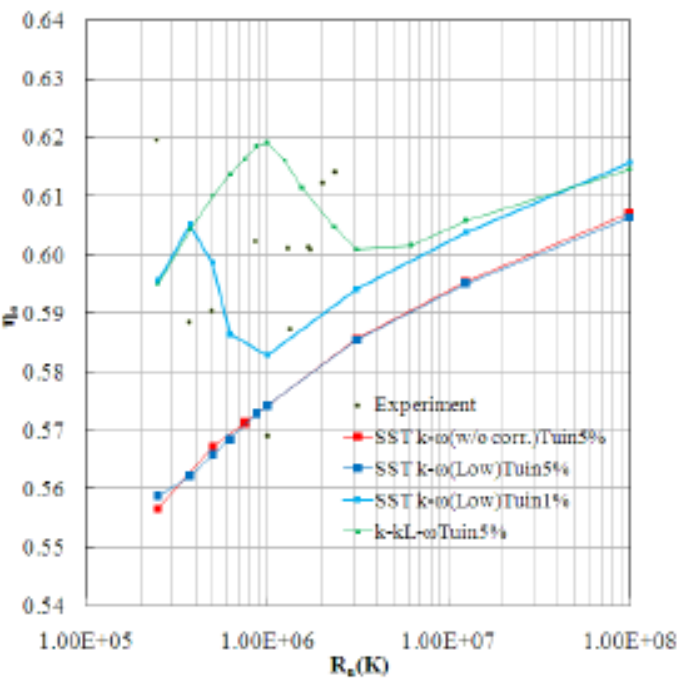

Figure 12: Effect of Reynolds number on $\eta_{\mathrm{o}}$

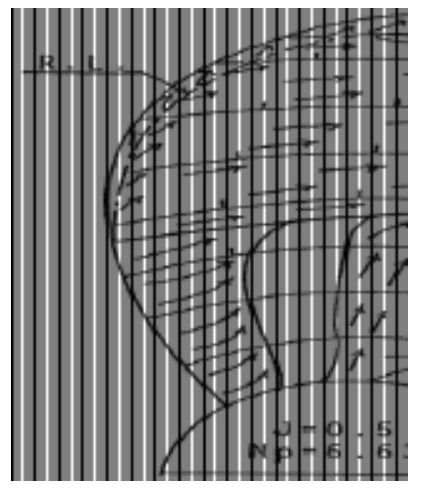

Figure 13: Oil flow visualization in the Experiment $\left(\mathrm{R}_{\mathrm{n}}(\mathrm{K})=6.75 \times 10^{5}\right)^{9}$

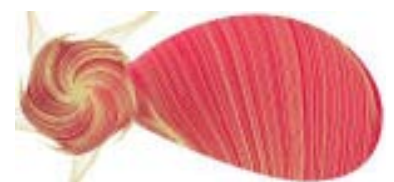

(a) $R_{n}(K)=2.5 \times 10^{5}$

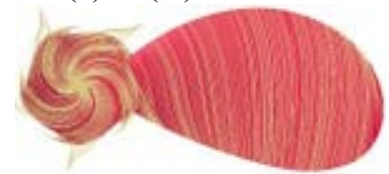

(b) $\mathrm{R}_{\mathrm{n}}(\mathrm{K})=1 \times 10^{6}$

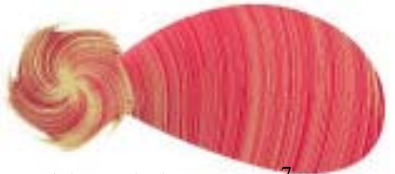

(c) $R_{n}(K)=1 \times 10^{7}$

SST k- $\omega$ (w/o corr.), $T_{\text {uin }} 5 \%$

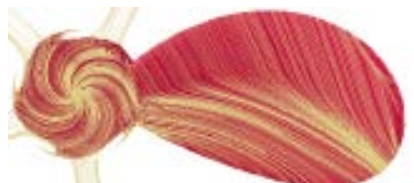

(a) $R_{n}(K)=2.5 \times 10^{5}$

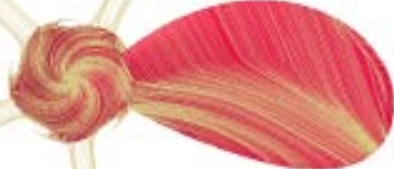

(b) $\mathrm{R}_{\mathrm{n}}(\mathrm{K})=3.7 \times 10^{5}$

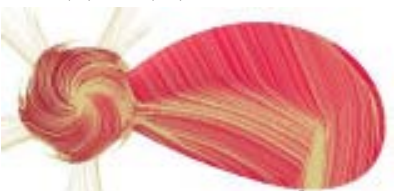

(c) $\mathrm{R}_{\mathrm{n}}(\mathrm{K})=5 \times 10^{5}$

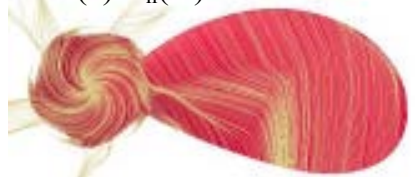

(d) $R_{n}(K)=6.2 \times 10^{5}$

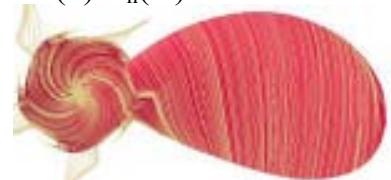

(e) $R_{n}(K)=1 \times 10^{6}$

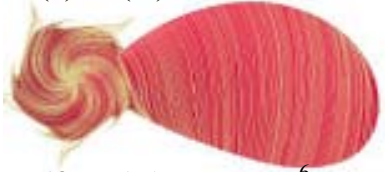

(f) $R_{n}(K)=3.1 \times 10^{6}$

SST k-w (Low), $\mathrm{T}_{\text {uin }} 1 \%$

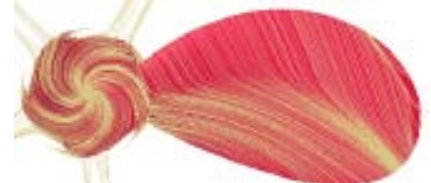

(a) $R_{n}(K)=2.5 \times 10^{5}$

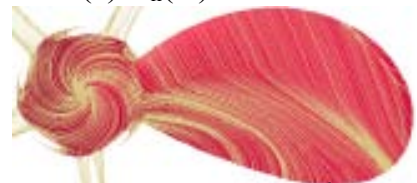

(b) $\mathrm{R}_{\mathrm{n}}(\mathrm{K})=1 \times 10^{6}$

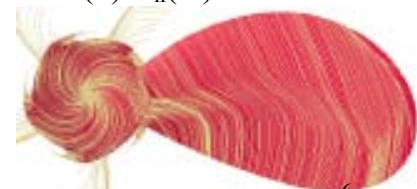

(c) $\mathrm{R}_{\mathrm{n}}(\mathrm{K})=1.5 \times 10^{6}$

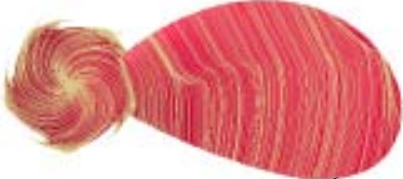

(d) $R_{n}(K)=2.3 \times 10^{6}$

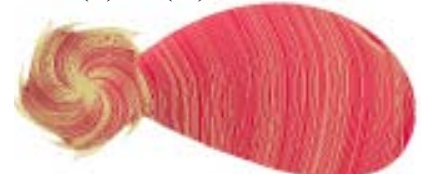

(e) $R_{n}(K)=3.1 \times 10^{6}$

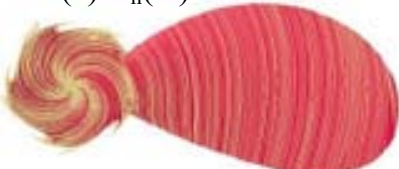

(f) $R_{n}(K)=1.2 \times 10^{7}$ $\mathrm{k}-\mathrm{k}_{\mathrm{L}}-\omega, \mathrm{T}_{\text {uin }} 5 \%$

Figure 14: Effect of Reynolds number on surface streamline 


\section{NUMERICAL SIMULATION OF CAVITATING FLOW}

\subsection{Experimental data of partially eroded propeller}

Cavitation performance test of a propeller for large bulk carrier was conducted. Principal particulars of model propeller and wake pattern are shown in Table 4 and Fig. 15. The wake distribution was simulated by wire mesh. High speed video observation was conducted at IHI Corporation R\&D Center (IIC in short). $\mathrm{K}_{\mathrm{T}}$ and $\sigma_{\mathrm{n}}$ were set to 0.16 and 1.68 respectively. Observation result is shown in Fig.16. Cavitation remained around trailing edge like a patch and collapse drastically at the $67^{\circ}$ in the rotating position. Paint erosion test was conducted at the University of Tokyo (UT in short). $\mathrm{K}_{\mathrm{T}}$ and $\sigma_{\mathrm{n}}$ were set to 0.16 and 1.53 respectively. Lower $\sigma_{\mathrm{n}}$ was applied for promoting cavitation erosion. Test result is shown in Fig. 17. Paint removal was confirmed at trailing edge near the tip.

Table 4 Principal particulars of model propellers (Yamasaki et al. 2011) ${ }^{10}$

\begin{tabular}{ll}
\hline Number of blades & 4 \\
Diameter & $241.8 \mathrm{~mm}$ \\
Pitch ratio & 0.69 \\
Expanded area ratio & 0.49 \\
Skew angle & $15^{\circ}$ \\
\hline
\end{tabular}

\subsection{Numerical evaluation of cavitation erosion risk}

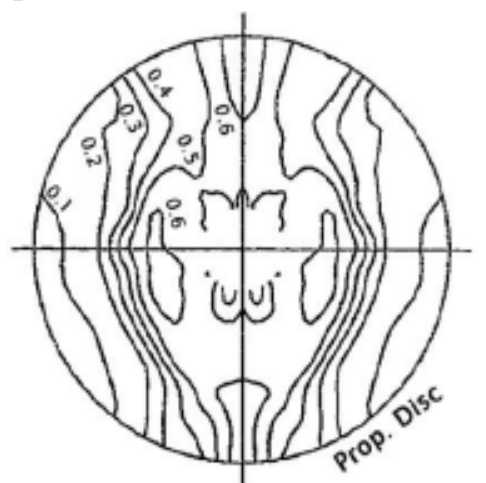

Figure 15: Wake patten ${ }^{10}$

Cavitation erosion is a fatigue phenomenon caused by the impact load generated when the cavitation bubble collapses acts repeatedly on the solid surface in neighborhood.

Nowadays, the generation of impact load is predictable in the state of the single bubble. However, flow analysis for actual turbo machinery needs to treat many bubbles collapse and needs to treat comparatively long time phenomenon. However, such detail simulation is not practical for actual design procedure in current computer ability.

Therefore, it is thought to be practicable that the physical index obtained from the global flow analysis is related to the strength of cavitation erosion. Nohmi et al. (2008) conducted the analysis of cavitating hydrofoil by the barotropy model in combination with the bubble dynamics, and suggested the simple four indexes ${ }^{11}$ related the strength of cavitation erosion from their finding.

In this research, one index of them was applied to the cavitating flow around propeller and the evaluation of the erosion risk was conducted. The index is shown in expression (10). Expression is the product of the numerical value of the cavity void fraction $\alpha$ at the surface position and the pressure recovery.

$$
\text { Index }=\alpha \cdot \max \left[\left(\mathrm{p}-\mathrm{p}_{\mathrm{v}}\right), 0\right]
$$

The adaptive mesh refinement methodology was used for the resolution of tip vortex. To 
calculate efficiently, the flow field around only one blade out of four blades was divided by fine meshes. This blade is called "Key blade" here and focused.

The propeller is rotated $1800^{\circ}$ by $1^{\circ}$ per cycle to develop flow field, after that $0.3^{\circ}$ per cycle was adopted during the final time step. The second order accuracy of the convective term was adapted. The full-cavitation model was used in the calculation. NCG was set to $1 \mathrm{ppm}$ and the empirical constants $C_{e}$ and $C_{c}$ were set to 0.02 and 0.01 respectively. $K_{T}$ and $\sigma_{n}$ were set to 0.16 and 1.68 respectively.

Simulated cavity extent (cavity void fraction $\alpha=0.1$ ) and contour of erosion index are shown in Fig. 18. Red color in contour map of erosion index means higher erosion risk.

In this case, cavitation remained like a patch at the $50-70^{\circ}$. This type of cavitation pattern is thought to be erosive. Simulated results show higher value of erosion index at the $55-65^{\circ}$ and correspond well with experimental results.

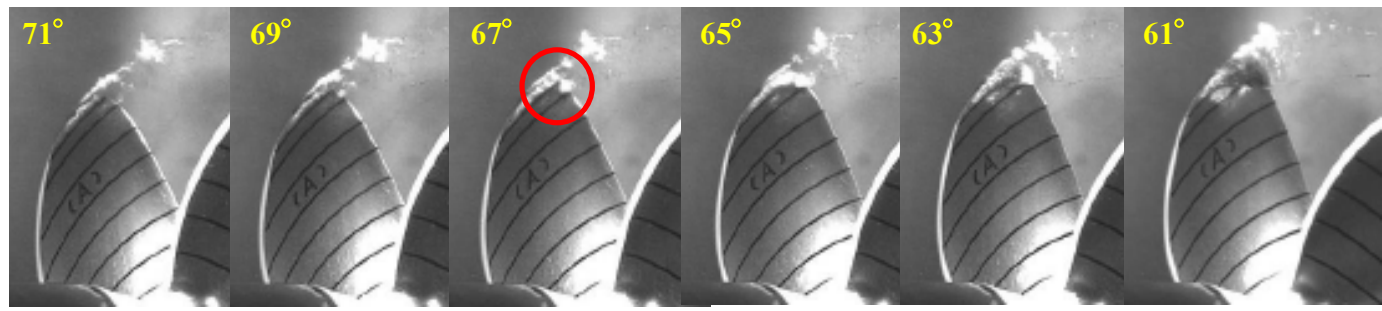

Figure 16: High speed video observation (IIC,Yamasaki et al.2011) ${ }^{10}$

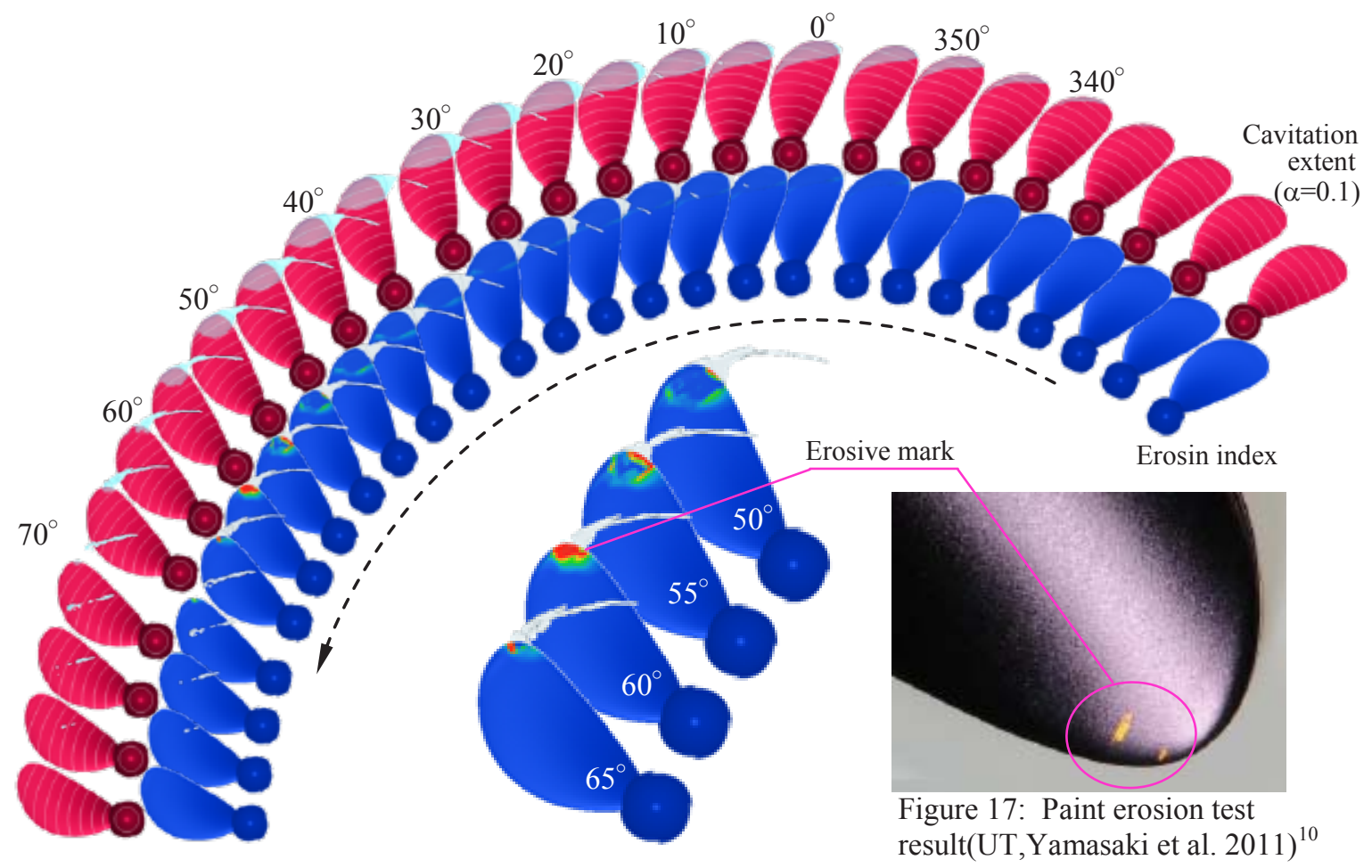

Fig. 18 Cavitation extent and contour of erosion index 


\subsection{Pressure pulse evaluation}

Pressure pulse at the propeller top position was measured at IIC as shown in Fig. 19. Pressure pulse amplitude is thought to be gratefully related to the second order time differential of cavity volume. In this research, pressure pulse and cavity volume variation were compared with experimental results in Fig. 20 and 21. Experimental pressure pulse was measured with transducer in flat plate. However, simulation were conducted without plate.

For considering this difference, illustrated pressure pulse in Fig.20 is product of simulated pressure and solid boundary factor 2 .

By using fine mesh for tip vortex resolution, higher order blade frequency component increased. Simulated pressure pulse wave agrees comparatively well with experimental results in this case.

On the other hand, calculated wave pattern of second order time differential of cavity volume was also corresponding well to experimental pattern. It was confirmed that pressure fluctuation was gratefully affected by second order time differential of cavity volume.

Authors also conducted the many simulations of other cases, simulated pressure pulse wave patterns were often quite different from pressure pulse measurement results, although the wave pattern of the second order time differential of cavity volume corresponded well to the experimental results.

In current situation, authors think that evaluation by using the second order time differential of cavity volume is available for improving cavitation performance.

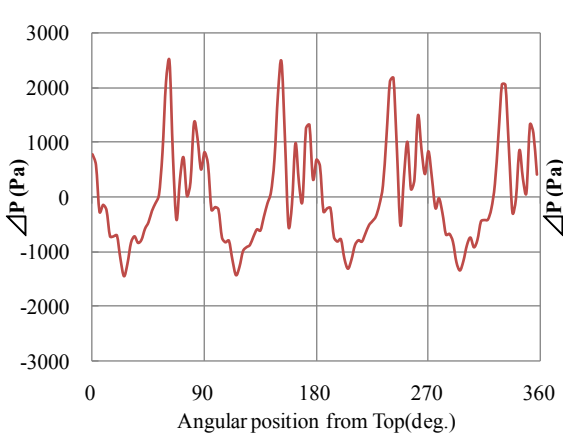

Fig. 19 Pressure pulse

(Experiment)(IIC,Yamasaki et al.2011) ${ }^{10}$

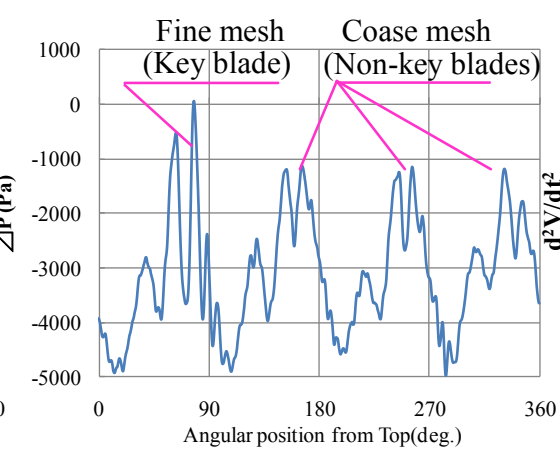

Fig. 20 Pressure pulse (Calculation)

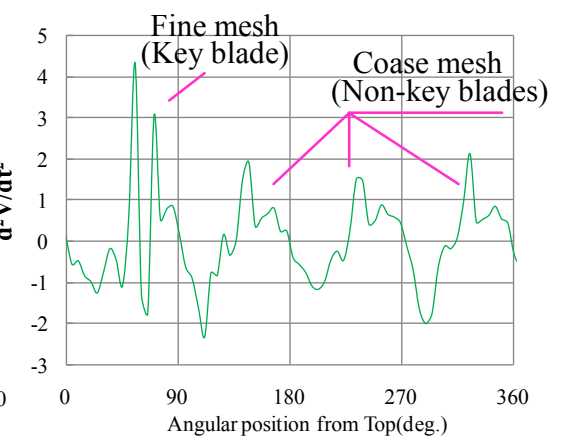

Fig. 21 Second order time differential of cavity volume(Calculation)

\section{CONCLUSIONS}

- The k- $\varepsilon$ model predicted the boundary layer thickness in good agreement with experimental results in the fully turbulent flow case.

- $\quad$ The SST k-w model underestimated the boundary layer thickness in model scale and predicted Reynolds number of transition to fully turbulent flow was lower than open water test tendency.

- The $\mathrm{k}-\mathrm{k}_{\mathrm{L}}-\omega$ model predicted boundary layer thickness and POC in comparatively good accuracy in the range of transitional Reynolds number.

- The cavitation simulation of the eroded propeller was conducted and erosion index was introduced. RANS predicted the erosive cavitation pattern. Predicted erosive 
position showed good agreement with experimental results.

- Cavitation simulation with tip vortex resolution is necessary for analyzing the higher order component of pressure pulse.

- Prediction accuracy of pressure pulse was not enough. Much improvement of discretizing accuracy is necessary. However, the evaluation by second order time differential of cavity volume gives valuable information for improving the pressure level of designing propeller.

\section{REFERENCES}

[1] Menter, F.,"Zonal Two Equation k- $\omega$ Turbulence Models for Aerodynamic Flows", AIAA 93-2906., (1993).

[2] Wilcox, D.,'Turbulence Modeling for CFD", Second Edition, CDW Industries, Inc., (1998).

[3] Walters, D.K. and Leylek, J.H.,"A New Model for Boundary Layer Transition Using a Single-Point RANS Approach", ASME Journal of Turbo machinery, 126, pp.193-202 (2004).

[4] Walters, D.K. and Cokljat, D.,"A Three-Equation Eddy-Viscosity Model for ReynoldsAveraged Navier-Stokes Simulations of Transitional Flow", ASME J. of Fluids Eng., 130, 121401 (2008).

[5] Singhal, A. K., Athavale, M. M., Li, H. and Jiang, Y., "Mathematical basis and validation of the full cavitation model", Journal of Fluids Engineering 124, pp.617-624 (2002).

[6] Gindroz, B., Hoshino, T. and Pylkkanen, V.,'Propeller RANS/Panel Method Workshop", 22nd ITTC Propulsion Committee Propeller RANS/Panel Method Workshop, Grenoble, Apr., (1998).

[7] Jessup, S.D.,"Experimental Data For RANS Calculations and Comparisons(DTMB P4119)", 22nd ITTC Propulsion Committee Propeller RANS/Panel Method Workshop, Grenoble, Apr., (1998).

[8] Funeno, I.,'On Viscous Flow around Marine Propellers - Hub Vortex and Scale Effect “, J. of Kansai SNA, Vol.238, pp.17-27, (2002).

[9] Takei, Y., Kakugawa, A. and Ukon, Y.,'Flow visualization on the blades of marine propellers", Flow Visualization, Vol.7, No.26, pp.7-10, (1987).

[10]Yamasaki, S., Hasuike, N., Himei, K., Okazaki, A., Mishima, T., Fujii, K., Yamaguchi, H., Kawanami, Y. and Ukon, Y.,"Cavitation Erosion of a Propeller for Large Bulk Carrier", Proc. of Conference Proceedings the Japan Society of Naval Architects and Ocean Engineers, Vol. 12, pp. 415-418,( 2011).

[11]Nohmi, M., Iga, Y. and Ikohagi, T.,'Numerical Prediction Method of Cavitation Erosion", Conference Proceeding of Turbomachinery Societh of Japan, vol.59, pp. 49-54,(2008). 